\title{
Comments on "Parameterization of the Autoconversion Process. Part I: Analytical Formulation of the Kessler-Type Parameterizations"
}

\author{
Robert Wood And Peter N. Blossey \\ University of Washington, Seattle, Washington
}

(Manuscript received 7 October 2004, in final form 14 January 2005)

In a recent paper, Liu and Daum (2004, hereafter LD04) derive analytical expressions for the rate of autoconversion of cloud droplets to embryonic raindrops for a range of assumptions about the dependence of the collection kernel upon cloud droplet size. Their analytical derivations are used to provide a physical formalism for some of the autoconversion parameterizations that are widely used in a broad range of numerical models (e.g., Kessler 1969; Tripoli and Cotton 1980; Baker 1993; Boucher et al. 1995).

The basis for LD04 is the integral [their Eq. (3)], here written in mass terms

$$
P=\int_{0}^{\infty}\left[\int_{0}^{\infty} K\left(x, x^{\prime}\right) x^{\prime} n\left(x^{\prime}\right) d x^{\prime}\right] n(x) d x,
$$

where $P$ is defined as the autoconversion rate in LD04, $x$ and $x^{\prime}$ are the masses of two coalescing droplets, $n(x)$ is the number of droplets in the mass range $x$ to $x+d x$, and $K\left(x, x^{\prime}\right)$ is the collection kernel for the coalescing droplets.

Most bulk precipitation schemes used in numerical models partition liquid water into separate cloud and rain classes using a separation particle radius $r_{0}$, or equivalently mass $x_{0}=\left(4 \pi \rho_{w} / 3\right) r_{0}^{3}$, to distinguish the classes. The choice of $r_{0}$ is taken to be $20 \mu \mathrm{m}$ in this paper. The autoconversion rate is then strictly defined as the rate at which liquid water is transferred, or equivalently converted, from the cloud class to the rain class by coalescence of two cloud droplets. For clarity, we term this the cloud-to-rain autoconversion rate (denoted here as $A$ ).

Corresponding author address: Dr. Robert Wood, Dept. of Atmospheric Sciences, University of Washington, Seattle, WA 98195.

E-mail: robwood@atmos.washington.edu
Autoconversion results from the collision of two cloud droplets with masses $x, x^{\prime}$ (each less than $x_{0}$ ) that form a rain droplet of mass $x+x^{\prime}>x_{0}$. Therefore, the autoconversion rate $A$ may be written (Beheng and Doms 1986) as

$$
A=\int_{0}^{x_{0}}\left[\int_{x_{0}-x}^{x_{0}} K\left(x, x^{\prime}\right) x^{\prime} n\left(x^{\prime}\right) d x^{\prime}\right] n(x) d x
$$

which can be derived directly from the stochastic collection equation assuming a symmetric kernel $K\left(x, x^{\prime}\right)$ $=K\left(x^{\prime}, x\right)$ (Beheng and Doms 1986). Note that the limits of integration in the inner integral reflect the constraint that $x+x^{\prime}>x_{0}$. Equation (2) gives the true cloud-to-rain autoconversion. Two cloud droplets with masses $x$ and $x^{\prime}$ can also coalesce to produce a second cloud droplet of mass $x+x^{\prime}<x_{0}$. This process is termed self-collection (Beheng and Doms 1986; Beheng 1994; Seifert and Beheng 2001), which here is given the symbol $S$. The sum of the autoconversion and self-collection of cloud droplets is the total rate of coalescence of cloud droplet mass.

Equations (1) and (2) are identical apart from the limits of integration, with the result that (2) is more difficult to solve in an analytic sense because the lower limit of the integral over $x^{\prime}$ is a function of $x$. Because of the different limits of integration (see Fig. 1), $P$ and $A$ have very different physical interpretations. Equation (1) represents the total rate of mass coalescence. In contrast, (2) is the rate of coalescence only of droplets that cross the $x_{0}$ separation mass. Clearly, $A$ is generally less than $P$ and represents the true autoconversion rate required by bulk parameterization schemes. While the use of thresholding criteria for autoconversion and the strong weighting of the collection kernel $K\left(x, x^{\prime}\right)$ toward larger $x$ and $x^{\prime}$ mimic to some degree the changes 

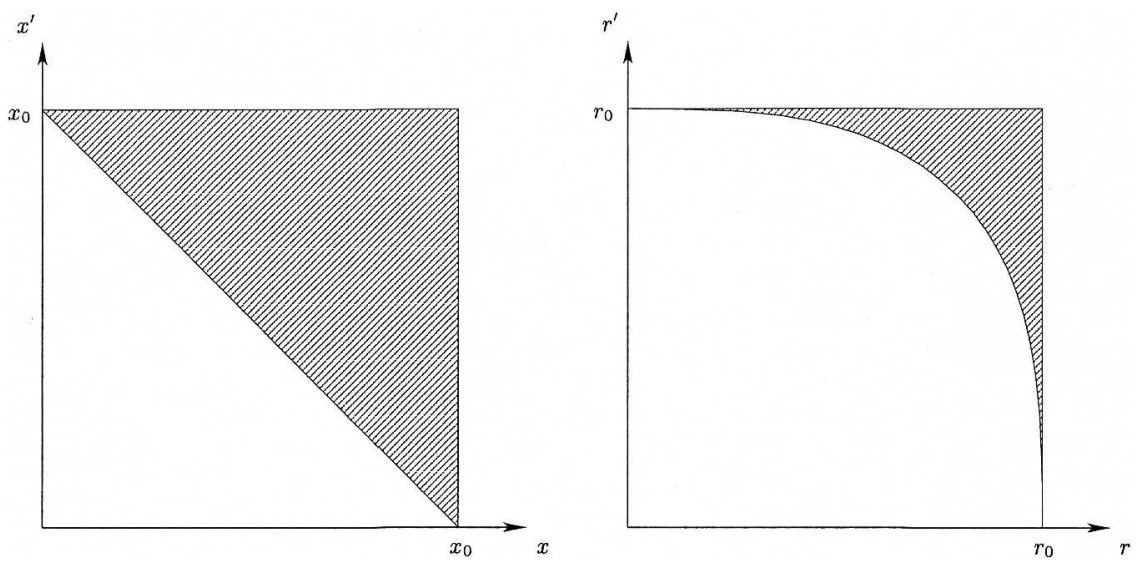

FIG. 1. The combinations of cloud droplets whose merging will yield a droplet whose (left) mass or (right) radius exceeds the separation $x_{0}$ or $r_{0}$, respectively. (left) The shaded region, $x+x^{\prime}>x_{0}$ and $x, x^{\prime}<x_{0}$, corresponds to the domain of integration in Eq. (2). (right) The shaded region corresponds to $r^{3}+r^{\prime 3}>r_{0}^{3}$ and $r, r^{\prime}<r_{0}$.

in the limits of integration from (1) to (2), the integral in Eq. (1) still overpredicts the true cloud-to-rain autoconversion substantially under realistic conditions, as is demonstrated below.

If we set the upper limits of integration to $x_{0}$ for both integrals in (1), we interpret the LD04 equation as being the total rate of mass coalescence of cloud $\left(r<r_{0}\right)$ droplets, a rate we label as $P_{x_{0}}$. Therefore, $P_{x_{0}}=A+S$, that is, the total mass coalescence is the sum of the self-collection and the autoconversion. We ask how different the values of $A$ and $P_{x_{0}}$ are for physically realistic cloud droplet size distributions and collection kernel $K\left(x, x^{\prime}\right)$. To achieve this we first use the collision kernel data of Hall (1980) and Stokes flow terminal velocity from Pruppacher and Klett (1997), assuming a coalescence efficiency of unity, to define $K\left(x, x^{\prime}\right)$. Our observational dataset comprises data from 12 flights of the U.K. Met Office C-130 in marine boundary layer (MBL) clouds [see Wood (2005a) for a complete description]. Droplet size distributions $(r<20 \mu \mathrm{m})$ are measured at a number of levels in each cloud. The cloud liquid water contents and droplet concentrations span the range commonly observed in stratiform MBL clouds.

For each of the size distributions in cloud, we derive $P_{x_{0}}$ and $A$ as described above. The integrals (1) and (2) are evaluated using the trapezoidal rule over logarithmically equi-spaced radius/mass bins. The results are shown in Fig. 2 and are in general agreement with the comparisons in Wood (2005b), where a time-dependent SCE solver was used to determine the cloud-to-rain autoconversion rates for the observed size distributions. We find that the 10th and 90th percentiles of $P_{x_{0}} / A$ are 3.8 and 112 with the median value being 9.3. Inclusion only of size distributions that satisfy the criti- cal radius threshold criterion of Liu et al. (2004) leads to a median value of $P_{x_{0}} / A$ equal to 7.3 with 10th and 90th percentiles of 3.2 and 15.7. Thus, the LD04 autoconversion rate $P$ results in an overprediction of the cloud-to-rain autoconversion rate by almost an order of magnitude even if the analytic LD04 bulk parameterization for the kernel $K\left(x, x^{\prime}\right)$ were perfect. This demonstrates that most of the mass coalescence of cloud droplets yields cloud droplets, that is, self-collection. Although we do not show it here, comparisons suggest that behavior of the LD04 bulk parameterization is qualitatively similar to $P_{x_{0}}$, overestimating $A$ by a factor of 2-10 (see Wood 2005b). This result is not restricted only to the parameterization derived in LD04, but would also apply to other parameterizations that are rooted in the evaluation of Eq. (1).

Figure 2b shows $P_{x_{0}} / A$ against the mean volume radius $r_{v}$. The dashed line indicates the results for a modified gamma distribution of cloud droplets (e.g., Austin et al. 1995) with the spectral width parameterized using Wood (2000). Only for spectra with $r_{v}$ close to $r_{0}$ does the LD04 integral (1) approximate the cloud-to-rain autoconversion rate.

One could argue, based upon the kinetic potential of nucleation theory developed by McGraw and Liu (2003) that the autoconversion rate should represent the mass transfer through coalescence across a separation radius that has physical significance, rather than one that is somewhat arbitrarily chosen to represent the size at which the importance of coalescence exceeds that of condensational growth. However, because the physically based separation radius is a complex function of cloud turbulence and thermodynamics (McGraw and Liu 2003), it is not possible to examine this with the 

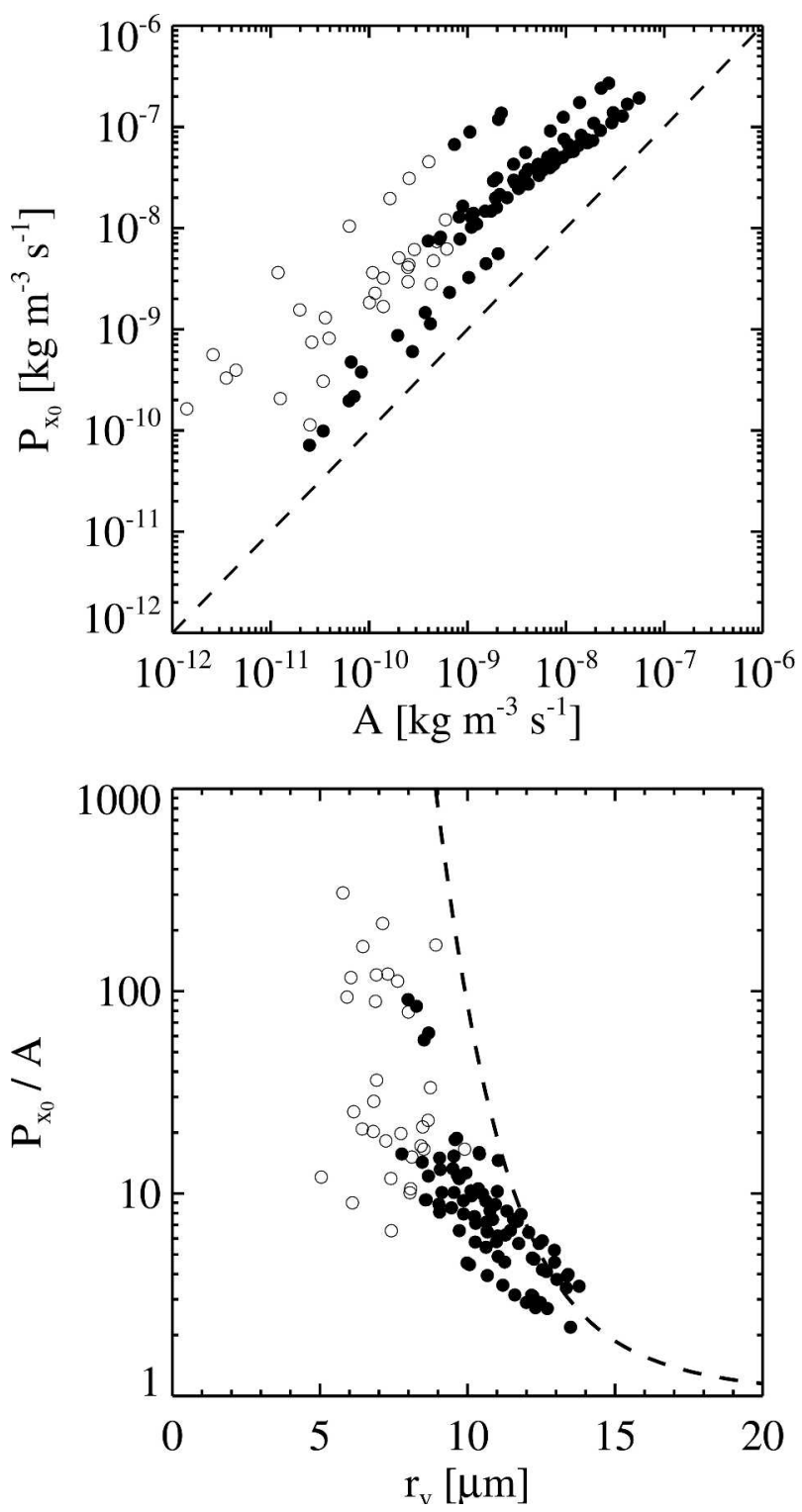

FIG. 2. (top) A plot of $P_{x_{0}}$ vs $A$ for spectra from aircraft flights. Open circles represent the subset of spectra that do not reach the sixth moment critical radius criterion for autoconversion in LD04; (bottom) the ratio $P_{x_{0}} / A$ versus mean volume radius $r_{v}$ for observations [circles, with open circles same as (top)]. Dashed line shows $P_{x_{0}} / A$ obtained assuming a modified gamma droplet size distribution (see text).

data we have available. However, we note that the physically based separation radius is almost always in the range $20<r<30 \mu \mathrm{m}$, and tests show that values of $r_{0}$ larger than $20 \mu \mathrm{m}$ result in even poorer agreement between $P_{x_{0}}$ and $A$.

To conclude, we have shown that the integral used in LD04 to determine the autoconversion rate does not give the cloud-to-rain autoconversion rate $A$; that is, the rate of transfer of mass across a particular radius separating cloud and rain classes by coalescence. Instead it gives the total rate of mass coalescence among cloud droplets and is typically much larger than $A$. We agree that the work of LD04 provides a solid theoretical basis for the Kessler-type parameterizations, which should prove fruitful in the quantitative determination of factors controlling warm rain production. However, we believe it important to note that this theoretical formulation has as its root an equation that can substantially overpredict the rate of conversion of mass from cloud droplets to embryonic raindrops as is commonly required by bulk precipitation schemes in numerical models.

Acknowledgments. The authors thank Drs. Liu and Daum for their insightful article, which casts new light on a difficult cloud microphysical problem, and for alerting us to the work of Dr. Beheng and his group. The authors wish to thank the staff of the Meteorological Research Flight and the C-130 aircrew and ground crew for their dedication in collecting the data presented in this study. We would also like to thank Chris Bretherton for helpful discussions.

\section{REFERENCES}

Austin, P., Y. Wang, R. Pincus, and V. Kujala, 1995: Precipitation in stratocumulus clouds: Observations and modeling results. J. Atmos. Sci., 52, 2329-2352.

Baker, M. B., 1993: Variability in concentrations of cloud condensation nuclei in the marine cloud-topped boundary layer. $\mathrm{Tel}$ lus, 45B, 458-472.

Beheng, K. D., 1994: A parameterization of warm cloud microphysical conversion processes. Atmos. Res., 33, 193-206.

_ , and G. Doms, 1986: A general formulation of collection rates of cloud and raindrops using the kinetic equation and comparison with parameterizations. Beitr. Phys. Atmos., 59, 66-84.

Boucher, O., H. LeTreut, and M. B. Baker, 1995: Precipitation and radiation modeling in a general circulation model: Introduction of cloud microphysical processes. J. Geophys. Res., 100, 16 395-16 414.

Hall, W. D., 1980: A detailed microphysical model within a twodimensional dynamic framework: Model description and preliminary results. J. Atmos. Sci., 37, 2486-2507.

Kessler, E., 1969: On the Distribution and Continuity of Water Substance in Atmospheric Circulation. Meteor. Monogr., No. 32, Amer. Meteor. Soc., 84 pp.

Liu, Y., and P. H. Daum, 2004: Parameterization of the autoconversion process. Part I: Analytical formulation of the Kesslertype parameterizations. J. Atmos. Sci., 61, 1539-1548.

- _ _ - and R. McGraw, 2004: An analytical expression for predicting the critical radius in the autoconversion parameterization. Geophys. Res. Lett., 31, L06121, doi:10.1029/ 2003 GL019117.

McGraw, R., and Y. Liu, 2003: Kinetic potential and barrier cross- 
ing: A model for warm cloud drizzle formation. Phys. Rev. Lett., 90.

Pruppacher, H. R., and J. D. Klett, 1997: Microphysics of Clouds and Precipitation. Kluwer Academic, 976 pp.

Seifert, A., and K. D. Beheng, 2001: A double-moment parameterization for simulating autoconversion, accretion and selfcollection. Atmos. Res., 59-60, 265-281.

Tripoli, G. J., and W. R. Cotton, 1980: A numerical investigation of several factors contributing to the observed variable den- sity of deep convection over south Florida. J. Appl. Meteor., 19, 1037-1063.

Wood, R., 2000: Parametrization of the effect of drizzle upon the droplet effective radius in stratocumulus clouds. Quart. J. Roy. Meteor. Soc., 126, 3309-3324.

, 2005a: Drizzle in stratiform boundary layer clouds. Part I: Vertical and horizontal structure. J. Atmos. Sci., in press. , 2005b: Drizzle in stratiform boundary layer clouds. Part II: Microphysical aspects. J. Atmos. Sci., in press. 